\title{
CONTEXTO DE CAMBIOS Y CONTINUIDADES EN EL PERÚ A FINALES DEL SIGLO XX. EL PAPEL DE LA CONFIEP, EN LA MODERNIZACIÓN DEL ESTADO.
}

\author{
Jorge Fuentes Dancourt \\ Universidad Ricardo Palma \\ betodancourt@gmail.com
}

\section{RESUMEN}

El presente trabajo de investigación será importante porque permitirá conocer, en qué medida el contexto histórico de la década de los '90, permitió impulsar las reformas políticas y económicas que posibilitan la crisis de crecimiento que registra el Perú actual. Nuestro objetivo será: Explorar [y conocer] en qué medida el contexto histórico de la década de los '90, impulso las reformas políticas y económicas que posibilitan la crisis del Perú actual. Nuestra hipótesis general de trabajo es: Si hay un crecimiento actual del Perú, se debió al funcionamiento razonable de las reformas políticas y económicas, que surgieron en el contexto de la década de los '90.

\section{PALABRAS CLAVE}

Reformas políticas / Década de los '90 / Modernización / Estado / CONFIEP.

\section{ABSTRACT}

This research is important because it reveals to what extent the historical context of the early ' 90 s, he helped boost political and economic reforms that enable the growth crisis that records the current Peru. Our goal is: To explore [and know] to what extent the historical context of the early '90s, I urge political and economic reforms that enable current crisis Peru. Our general hypothesis is: If there is a current growth of Peru, was due to the reasonable functioning of political and economic reforms, which arose in the context of the ' $90 \mathrm{~s}$.

\section{KEYWORDS}

Political reforms / Early 90s / Modernization / State / CONFIEP. 
Si el contexto histórico de los noventa es de crisis entonces mayor la necesidad de aceptar las reformas políticas y económicas propuestas por los empresarios representados entre otros por CONFIEP.

Asumiendo la responsabilidad de sus retos y desafíos, además demostrando madures y fortaleza política al estar representado al Congreso Constituyente Democrático, CCD, en los diversos grupos políticos que se presentaron, manifestando capacidad de gestión al impulsar con éxito una constitución liberal que diera las bases ideológicas para las reformas políticas necesarias y emprender el cambio, a través de las reformas del Estado y las reformas económicas.

Encontrando en la coyuntura, interna y externa, la oportunidad y escusa necesaria para gestionar los cambios, ajustados a sus necesidades, demostrando fortaleza política y empresarial lo que refleja su madures al frente del devenir histórico del Perú; y a sus necesidades de clase.

\section{MARCO TEÓRICO}

La presente investigación se encuentra imbuida en el debate historiográfico de la Historia inmediata o también llamada Historia del Presente, o Historia Actual.

Siendo la formulación del problema, “¿En qué medida el contexto histórico de la década de los '90, impulso las reformas políticas y económicas qué posibilitan la crisis de crecimiento del Perú actual?

Los que han informado, analizado y argumentado de ¿Qué paso en la susodicha década? son los periodistas, abogados, economistas, sociólogos entre otros científicos sociales, pero muy pocos historiadores.

A menudo se dice que los periodistas brindan el "primer borrador de la historia". Ello hace pensar a priori que los historiadores intervienen posteriormente $\mathrm{y}$, basándose en una variedad de fuentes, revisan ese "primer borrador" para convertirlo en algo más elaborado. Pero ya que los historiadores no abordan los acontecimientos de la misma manera que los periodistas, algún historiador tendría que ser el primero en escribir dicho "primer borrador". Ya que se percibe cierta necesidad o urgencia por analizar los eventos contemporáneos. 
La tarea del historiador tradicional ha sido traer de vuelta al presente un pasado "muerto" "que ya no está" que está ausente. Tal vez, aquellos que escriben sobre historia reciente reconocen que su sujeto de estudio no es todo el pasado, o como lo señala Renee Romano, sobre uno que "aún no está muerto".

Por todo esto, escribir historia contemporánea también ofrece numerosas oportunidades. Quienes escribimos sobre los hechos recientes podemos tener la oportunidad de redactar el "primer borrador" que servirá de punto de partida para la historiografía posterior.

Lo acontecido en la década de los '90 son hechos que podemos observar en el día a día a través de los medios de comunicación; para la Historia Tradicional, son los hechos, lo importante, descritos a través del tiempo y que se encuentran en las fuentes escritas, y que deberíamos hacer un inventario de estas fuentes documentales elaborando una crítica de documental de ellas. Al finalizar con estas tareas se debían realizar las operaciones sintéticas en varias etapas ${ }^{1}$ :

1. Comparar los documentos para establecer un hecho particular.

2. Reagrupar los hechos generales en marcos generales.

3. Manejar el razonamiento, sea por deducción o por analogía para relacionar los hechos entre sí y llenar los vacíos.

4. Obligar a practicar una elección entre la masa de acontecimientos $\mathrm{y}$

5. Llevar al historiador a intentar algunas generalizaciones $\mathrm{y}$ arriesgarse a algunas interpretaciones.

Para la historiografía del siglo XX, en particular para los Annales la historia debe interesarse por las sociedades humanas en el tiempo y en el espacio y no como se afirmaba corrientemente que su objeto de estudio era solo los hechos del pasado, en ese punto estamos, pues queremos comprender la relación entre la coyuntura vivida en la década de los ' 90 tanto en el interior del Perú como en el mundo, y como ésta afecto de manera directa a los problemas y decisiones que afrontamos; además como este espacio cada vez mas interconectado, globalizado influye para bien o para mal, en nuestro contexto histórico, interrelacionando el

1 BOURDÉ, Guy y Hervé MARTIN, Les écoleshistoriques, pp. 148-149 
pasado y el presente, además valorar los aportes de las otras disciplinas siendo interdisciplinarios o multidisciplinarios para integrarlas al discurso histórico .

Ahora sobre el concepto "crisis" es un instrumento teórico de primer orden que está profundamente ligado a la historia ${ }^{2}$; para Fernando Rosas la idea de crisis está ligada:

En ese sentido, la noción de crisis es otro de los conceptos que -estando profundamente ligada a la historia- es tomada, desde hace relativamente poco tiempo, como una herramienta teórica de primer orden para conocer determinados períodos históricos en donde diversos indicadores materiales y mentalesevidencian fluctuaciones particularmente intensas, alterando los niveles de vida de la sociedad en su conjunto.

\section{ANÁLISIS DEL CONTEXTO HISTÓRICO DEL SISTEMA DE GO- BIERNO, SISTEMA ELECTORAL Y SISTEMAS DE PARTIDOS POLÍTICOS EN EL CONTEXTO DE LA DÉCADA DE LOS '90.}

Se enmarca dentro del proceso histórico peruano de los últimos años en dos vías que se complementan lo interno y lo externo; en lo primero es la modernización del Estado, propiciado, recomendado y planteado por muchos en el país, para nosotros el papel fundamental que tiene la Confederación Nacional de Instituciones Empresariales Privadas; en lo segundo es el plano internacional la Globalización.

La modernización del Estado, reducción del Estado, reorganización del estado, reestructuración de Estado, son algunos de los términos que en los últimos 40 años se tienen utilizados para modificar al Estado o para desaparecer por ley los órganos que constituyen el Estado Peruano.

En la década de los noventa, en medio de un contexto internacional marcado por la globalización y el neo liberalismo planteado por las potencias se da un giro importante en el Perú, se aprueba una nueva constitución política la de 1993, y esto deviene en una corriente modernista que privatizó todo lo que el Estado poseía.

2 ROSAS, Fernando. "Crisis e historia: algunas consideraciones sobre la economía de Europa Occidental en los siglos XIV y XVII" En: Sociedad y cambio en Occidente. Lima: Universidad de Lima. 2002 
Los gobiernos de los últimos 40 años, recordemos que Juan Velazco Alvarado, Francisco Morales Bermúdez, Fernando Belaunde Terry, Alan García Pérez y Alberto Fujimori, fueron los gobernantes que fragmentaron al Estado, unos más que otros; fueron los que deshicieron el Estado y que no contribuyeron a fortalecerlo, modernizarlo y mucho menos ponerlo al servicio de los intereses del país.

En esta modernización juega un papel importante la CONFIEP Confederación Nacional de Instituciones Empresariales Privadas; que piensa sobre la necesidad de mejorar la gestión (gerencia) del Estado, según estos los problemas son el sobredimensionamiento, ineficiencia, despilfarro de recursos, entre otros, sin embargo poco se ha hecho por enmendar estos problemas.

En opinión de la CONFIEP, cualquier reforma del Estado debe rescatar los principios fundamentales que deben guiar la gestión de un Estado al servicio de sus ciudadanos. Según estos, es urgente recuperar la noción de que la razón de ser del Estado es el ciudadano: existe para servirlo.

En la década de los'80, con el retorno a la Democracia gobernó el Perú, don Fernando Belaunde Terry de 1980 a 1985; luego el APRA por primera vez en su historia llega a la presidencia con Alan García Pérez, quien gobernó de 1985 a 1990; esta década transcurrió con graves problemas, en lo económico una creciente inflación que a finales de la década muto en híper inflación, y esto se tradujo en una alza incesante del coste de vida para la población, aunado a ello la caída del PBI, con el alejamiento de los organismos económicos internacionales, como por ejemplo el FMI, que nos consideró Inelegibles (que no éramos sujetos de crédito, por el no pago de la deuda que en este contexto era enorme e impagable), el Banco Mundial, el Club de Paris. Por otro lado el malestar en torno a lo económico era grave, el sindicato de trabajadores cada vez más fraccionados o desestructurados, reclamando mayores aumentos de salarios y condiciones de vida, traducida en paros, manifestaciones, etc.; por el otro los sindicatos de empresarios llamase CONFIEP, SIN, SNM, ADEX, entre otros cada vez más unidos, reclamando cambios radicales en la manera de la conducción de las políticas económicas, a finales de la década se le debe agregar un desatino mayor la estatización de los bancos, que llevo a que los empresarios se unan de manera definitiva creando una plataforma política alternativa al gobierno y contestataria a la sociedad, llamado "Movimiento 
Libertad" y conducido por el prestigioso literato Mario Vargas Llosa, quien era a su vez propulsor de la vieja ideología económica pero con matices nuevos llamado "Neoliberalismo", que estaba muy de boga en el primer mundo; en lo político una crisis del Estado en proporciones nunca vista, su ineficacia para resolver los problemas económicos se tradujo en resquebrajamiento de la estructura familiar que los llevó a un estado de sobrevivencia tal que aumentó considerablemente la delincuencia, la prostitución, el narcotráfico; o como lo manifiesta Matos Mar, "formas violentas de obtención de recursos o ingresos" (1984:63); en paralelo esta década está marcada por Sendero Luminoso y su líder Abimael Guzmán, que marcaría el fraccionamiento de la sociedad, y el incremento de la violencia a niveles destructivos en las cuales el Estado también muestra su ineptitud para resolver un problema histórico entre el Perú formal y el real, no basto decir que eran genocidas para poder condenarlos como terroristas a cadena perpetua, era y es, un problema en la estructura histórica del Perú que se inicia en los albores de la independencia, cuando se funda el concepto de Estado y ciudadanía. En lo social hay un crecimiento urbano acelerado y a su vez desordenado e informal, dando un nuevo rostro a las ciudades costeñas en especial a Lima, por la migración del campo a la ciudad. No es raro que la producción agrícola cayera a niveles de la década de los 60 eso se debe a dos puntos, el primero la Reforma Agraria, que generó tantos problemas y que hasta el presente no han sido resueltos y por otro lado esa masa campesina liberada del yugo gamonal o de la tierra busco nuevos y mejores horizontes, produciéndose una masiva migración del campo a las ciudades costeñas, generando una serie de cambios.

Este sería en resumen el contexto a finales de la década de 1980, que llegaría con un cambio democrático de alternancia política.

Las elecciones generales y democráticas, se llevaron a cabo en abril 08 y en junio 10 de 1990, renovando el Poder Legislativo y Ejecutivo del Perú.

Los candidatos a la presidencia fueron:

- FREDEMO, Mario Vargas Llosa.

- APRA, Luis Alva Castro.

- CAMBIO 90, Alberto Fujimori Fujimori.

- Izquierda Unida - IU, Henry Pease García. 
- Izquierda Socialista, Alfonso Barrantes Lingán.

- FRENATRACA, Roger Cáceres Velásquez.

- Entre otros.

El FREDEMO - Frente Democrático, era la coalición de tres partidos políticos importantes, el primero "Movimiento Libertad" encabezado por Mario Vargas Llosa, el PPC (Partido Popular Cristiano) dirigido por Luis bedoya Reyes, y Acción Popular encabezado por Fernando Belaunde Terry.

El APRA, encabezado por Luis Alva Castro.

CAMBIO 90 de Alberto Fujimori; eran los tres movimientos más importantes en estas elecciones, ninguno alcanzo la mayoría solicitada $50 \%+1$ de los votos válidos emitidos y pasaron a la segunda vuelta el Frente Democrático y Cambio 90. Los resultados fueron así (cuadro 1)

La segunda vuelta se realizaría el 08 de junio de 1990, y los resultados serían así:
$62.4 \%$
Alberto Fujimori
Cambio 90
$37.6 \%$ Mario Vargas Llosa
FREDEMO

Cuadro 1

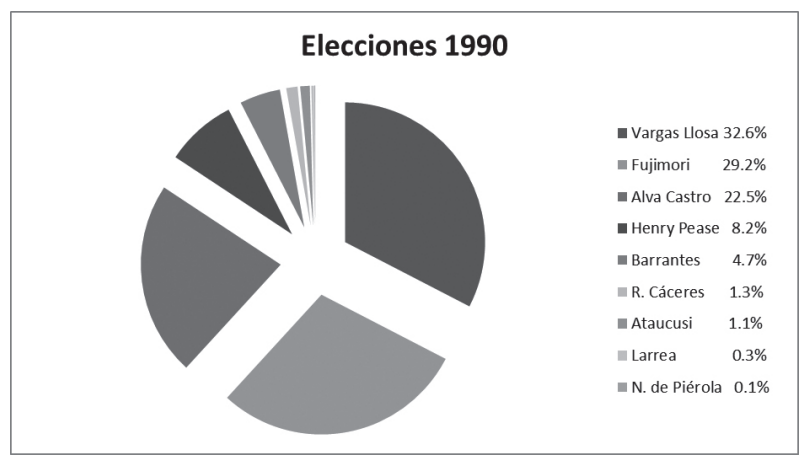

Los resultados en el Poder Legislativo fueron: 
Cuadro 2

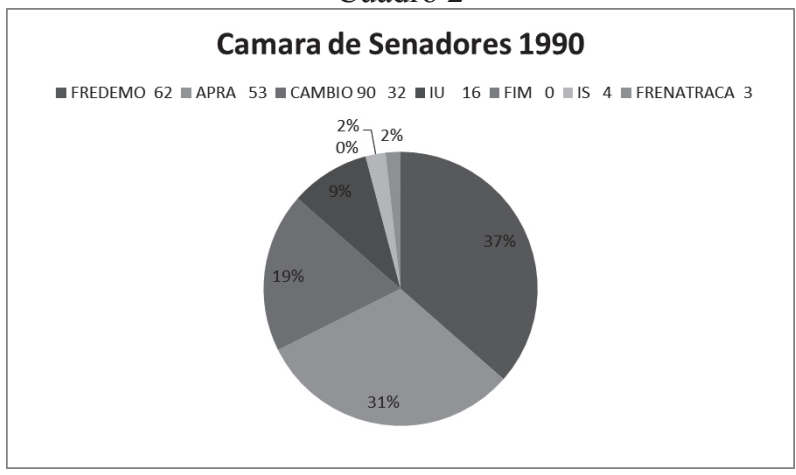

Cuadro 3

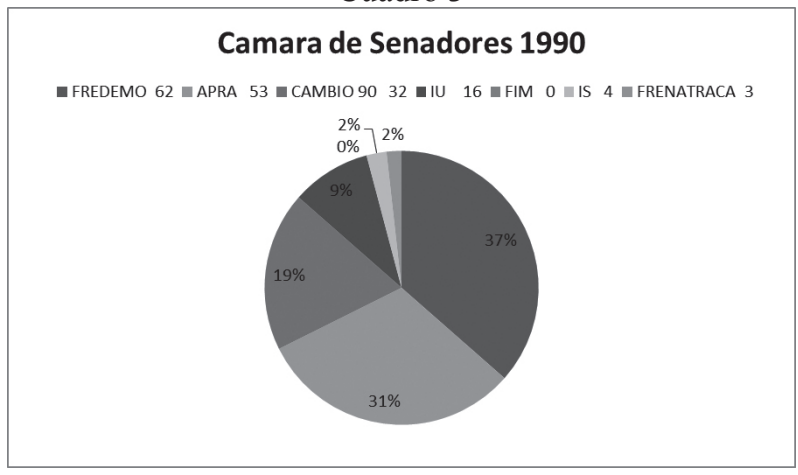

Para Henry Pease, con Fujimori se inicia el Estado Neoliberal (2013, p. 326), en efecto por consecuencia de la híper inflación, la caída estrepitosa del PBI, y la paralización de la inversión privada, sumado a esto que el partido ganador de las elecciones Cambio 90 de Alberto Fujimori considerado un outsider, no tiene plan de gobierno (eso es una constante en los llamados outsider, llegan al poder por un discurso motivador a los oídos de las masas, que los ve como los mesías o salvadores, sin siquiera plantear un plan básico de acción política y económica), por tanto asume o recoge o se le juntan los mismo asesores y políticos que estuvieron en la orilla de al frente, es decir del Frente Democrático y al interior de ellos, las ideas del Movimiento Libertad, fue tanto el descaro que el líder del 
movimiento, el escritor Mario Vargas Llosa se retira del Perú, muy dolido y en España donde fija su residencia, renuncia a la ciudadanía peruana y se hace ciudadano del mundo, entendiendo claramente el nuevo orden mundial que se avecinaba "la Globalización".

Primero plantea un ajuste económico llamado "shock", que no es otra cosa que un alza desmedida de los productos de la canasta básica a niveles astronómicos dícese para frenar la híper inflación pero lo que no dijeron era que el pueblo pago por este ajuste económico que aun hasta la fecha no se supera.

Por otro lado se fractura el orden democrático mediante un auto golpe y el cierre del Congreso dando origen a un régimen político autoritario y personalista que se hizo llamar pragmático (que no es otra cosa que una manera práctica (fácil, pensando que el resto es "politiquería barata") de hacer las cosas, buscando las consecuencias prácticas del pensamiento imponiendo su criterio de verdad en su eficiencia y valor para la vida política), no político o negando la política tradicional, según él enredada e ineficiente. Que fue aprobado por una gran parte de la sociedad que se vio reflejada por las encuestas que sospechosa e inmediatamente se hicieron públicas, lo que le permitió al régimen obtener legitimidad social tanto interna como externa producto de la coyuntura internacional luego de la caída del Muro de Berlín y el fin del comunismo.

Al imponer un nuevo orden constitucional a través de la Constitución Política del Perú de 1993, aprobada por referéndum, establece un Estado pequeño, producto de las privatizaciones y el escaso presupuesto que les asigna a varias instituciones públicas (como la Policía Nacional del Perú, los gastos en educación y salud, entre otros) que luego perjudicarían al propios país por su pobre rendimiento, unitario que se mantenía igual que en la constitución de 1979 y su predecesora de 1933, el Perú es un Estado Unitario pues la otra alternativa seria ser federal y nuestro país no está organizado de tal manera como si lo están México, Argentina y los Estados Unidos de Norteamérica, y de baja capacidad de gestión, básicamente por el descredito que significaba trabajar para el Estado y las magras remuneraciones que hacían inviables el ingreso de mejores y más capacitados profesionales.

En 1990 se da inicio a un Estado Neoliberal, brutal, despiadado sobre todo con las clases menos pudientes, donde se plantea la no intervención 
estatal, y la libertad absoluta del mercado, llevándose a la poca industria sobreviviente de la década de 1980, y junto con ella desapareció los sindicatos industriales además, empezó la lucha contra los sindicatos sobrevivientes. Donde Gonzales de Olarte menciona como rápidamente se destruye el viejo orden, en oposición al Régimen Velasquista de los '70 (1998, p.33).

Esto fue llevado a cabo por aquellos grupos políticos económicos como los empresarios agrupados en torno a la Confederación Nacional de Instituciones Empresariales Privadas - La CONFIEP es la institución que congrega y representa a la actividad empresarial privada peruana, a nivel nacional e internacional. Su principal objetivo es contribuir con el proceso de crecimiento económico sostenido, basado en inversión y fomento del empleo, a partir del esfuerzo de la iniciativa individual, la difusión de la empresa y la propiedad privada. Tiene como responsabilidad creciente examinar el contexto mundial y promover la integración del país en los mercados internacionales.

Para Pease estos grupos empresariales primero estuvieron con Velasco, luego con Belaunde y después con García, a través de los 12 apóstoles que al final se aliaron con el fujimorismo, (2013: 334) aquí lo más resaltante es como estos grupos empresariales lograron al final plasmar sus ideales políticos - económicos en la sociedad,

La privatización de las empresas públicas, como por ejemplo la Compañía Peruana de Teléfonos comprada por Telefónica de España y que se llama hasta hoy Telefónica del Perú, y que además de ser privatizada se le dio y promulgo, una ley de inversión mediante la cual se le exonera de pago por Impuesto a la Renta de Tercera Categoría, y por lo cual sus ganancias se elevaron exponencialmente; y como estas muchas otras empresas del Estado corrieron la misma suerte, muchas de ellas se vendieron de manera subvaluada. En paralelo según Pease el amplio armazón legal establecido para proteger a los trabajadores fue destruido, (2013, p.337).

Los organismos financieros internacionales, condicionaron su ayuda a los países del Tercer Mundo, como el Fondo Monetario Internacional (FMI), el Banco Mundial (BM), el Club de París, argumentando que las ultimas décadas el Estado de bienestar o interventor impuesto después de la Gran Depresión de los años '30, ha fracasado y por tanto llegado a su fin, en América Latina Chile es el primer Estado en adoptar las medidas 
Neoliberales, seguido de Argentina. Mediante este liberalismo aplicado a la economía aseguraba un mercado libre, producía más autonomía dícese para el individuo en este caso el empresariado; este liberalismo político y económico no fue aplicado a una justicia social que según los cultores del liberalismo es pura demagogia.

En este contexto se empieza a aplicar en el Perú lo que se llamó como "Consenso de Washington", que no es otra cosa que un conjunto de medidas que se aplicaron el América Latina y utilizadas por las instituciones multilaterales como el FMI, el BM, el BID, entre otros con el fin de salvaguardar las medidas liberales recién implementadas, entre estas tenemos:

- Disciplina fiscal

- Reducción del gasto público, tanto en educación como en salud.

- Reforma tributaria.

- Tasas de interés fijadas por el mercado.

- Tasas de cambio competitivas.

- Desbloqueo de la política comercial.

- Liberalización de la inversión extrajera.

- Privatización de las empresas públicas.

- Desregularización para impulsare la competencia.

- Seguridad legal para los derechos de propiedad.

Para Henry Pease se puede concluir que el estado neoliberal fue una respuesta pendular al Estado intervencionista instaurado por Velasco en la década de los '70 (2013, p.334)

Con los partidos políticos en descredito producto de la crisis de la década de 1980, todo como producto de la crisis de representación política, que desencadeno una crisis política sin precedentes, le siguió, una cultura política sin debates o discusiones en tanto el régimen político autoritario y personalista imponía sus ideas contra los partidos políticos tradicionales que fueron apabullados en los años subsiguientes, no habiendo una respuesta orgánica de parte de los actores políticos donde su función principal es canalizar las demandas de todos los sectores de la sociedad pero que a 
partir de la década de 1990 solo perciben y canalizan las demandas de un solo sector social, los empresarios.

En teoría los partidos políticos son organizaciones que buscan alcanzar el poder mediante el voto popular en los comicios pero a su vez tienen el objetivo de canalizar las demandas de los distintos sectores de la sociedad, llámese por ejemplo los grupos empresariales por un lado y los gremios organizados en sindicatos de trabajadores por el otro; estos grupos políticos deberán implementar intereses políticos partidarios, siendo los partidos políticos sujetos al cambio histórico pues el contexto histórico en el que surgen es distinto cada cierto tiempo.

En el Perú en la década de 1990, los partidos políticos son organizaciones que buscan establecer entre ellos una relación ya sea de oposición como el APRA, y otros de cooperación, como el Partido Popular Cristiano PPC, el Frente Independiente Moralizador, FMI, de Fernando Olivera, el Partido de Renovación Nacional de Rafael Rey Rey, el Movimiento Democrático de Izquierda, de Henry Pease y la Coordinadora Democrática CODE.

Estos se pueden clasificar políticamente en: el APRA para la década de los 1990 es un partido que bajo el liderazgo político de Alan García Pérez se posiciona en el centro cada vez más hacia la derecha. El partido Popular Cristiano que dirige Luis Bedoya Reyes, aunque no lo aceptan es un partido de derecha que apoyo rápida y muy comprometido con las reformas estructurales que se hicieron. Los demás son agrupaciones políticas que surgieron en el furor de los cambios pero que no lograron consolidarse, más fueron relevantes para la coalición, el coerción o chantaje político por el cual se obtienen favores o prebendas y legitimar las reformas.

En ese plano las características de los partidos políticos, se implantan en el Perú con el único objetivo de tener una plataforma electoral que les sirva a sus interés que son llegar al poder, teniendo una estructura organizativa donde el líder del movimiento político tiene la toma de decisiones y donde no existe la democracia interna, o si existe es una speudodemocracia interna

En el sistema electoral siguiendo los ideas de Jaime Guevara de UNAM, 2004, es el conjunto de medios a través de los cuales la voluntad de los ciudadanos se transformó en órganos de gobierno o de representación política. 
En el Perú según el artículo 177 de la CPP de 1993, el Jurado Nacional de Elecciones (JNE), es el órgano que administra justicia en materia electoral, fiscaliza los procesos electorales. Junto a ella se encuentra la Oficina Nacional de Procesos Electorales (ONPE), que se encarga de organizar, planificar y ejecutar los procesos electorales en el Perú. Y el Registro Nacional de identificación y Estado civil (RENIEC)

Analizando el contexto histórico planetario, en la década de los '90 el mundo se ve envuelto en una serie de acontecimientos, que marcan el desenlace de un siglo: se podrían enumerar un sinfín de situaciones como, el Fin de la guerra Fría; la Caída del Muro de Berlín; el Mundo Unipolar; el apogeo de las PC (computador personal, hardware); el aparecimiento del Windows que es un software que potenció las PC, a un nivel nunca antes visto a esto debe agregar el INTERNET; el surgimiento de un nuevo orden económico mundial el Neoliberalismo, impuesto básicamente por los líderes mundiales más representativos de aquellos días la Sra. Margaret Thatcher y Ronald Reagan, que pusieron fin al Estado benefactor, o como otros les llaman la desregularización de la economía. Todo esto dio origen a un nuevo orden mundial, al que se denomina Globalización.

El término "globalización", propone la unicidad del mundo, del planeta, es "la aldea global", es además un fenómeno económico donde se presenta mayor interdependencia entre los países, como lo indicaba Jean Claude Lavigne, en su texto La Globalización menciona que “... es más que la estructuralización mundial de las economías con relación a los principios liberales: un mecanismo de creación mundial, hecho de interconexiones, no solamente de intercambios, sino también de finanzas, de cultura, de información y de modos de vida. Este sistema concede a considerar el mundo como un solo espacio, en la cual puede ser tomada las decisión que permitan optimizar el provecho (búsqueda de los salarios más bajos, o las mayores ventajas fiscales, etc.)"

Esta globalización es un hecho histórico, es un término muy usado a partir de los '90, en especial luego de varios sucesos acaecidos en esa década como la caída del Muro de Berlín, el fin del Comunismo, el término de la Guerra Fría, con el triunfo del Capitalismo encabezado por los Estados Unidos, con el resurgimiento del liberalismo ahora llamado Neoliberalismo, masificación de los ordenadores personales (PC) y los software como el Internet, el Office, entre otros, aunque esta globalización o unicidad o globalización geográfica que AlvinToffler, en su texto, La 
Tercera Ola, plantea que tiene sus raíces en el descubrimiento de América. Es decir hace 519 años, el gestor de ello fue Cristóbal Colon, al que le tomo dos meses llegar desde puerto de Cádiz. Esto confirmó que el planeta era una esfera, un globo terráqueo; y este fue un hecho geográfico, que transformo las estructuras sociales, económicas y culturales del mundo europeo y mundial, además de consolidar un proceso económico que se venía dando en Europa, pero acaso a esto se le puede llamar el inicio de la globalización.

En el contexto histórico de la globalización, hay un hecho muy importante que debemos tomar en cuenta, la Segunda Revolución Industrial de mediados del siglo XIX, que se expande a todo el territorio europeo, norteamericano, y a finales del siglo al Japón; haciendo y vendiendo el desarrollo al mundo, como una teoría europacentrista, este proceso económico generó, a través del descentralismo económico el rompimiento de la unidad territorial. Esto simbolizo, el desinterés en la interacción de los pueblos y la transmisión cultural, como las ideas de unificación a través de la educación con ello las ideas nacionalistas se adoptaron al trabajo asalariado.

Con ello se dieron ideas subterráneas del cosmopolitismo lo cual generó: la competencia de los países industrializados por la posesión de las materias primas. Que le garantizaran el sostenimiento de sus industrias; y el entrelazamiento de las culturas, implantando tradiciones homogenizantes.

La globalización, es entonces una nueva fase del capitalismo, para Eric Hobsbawm; está asociado con el "capitalismo financiero", que se concreta con una serie de sucesos y a ello se le debe sumar el desarrollo tecnológico en las comunicaciones llamado "Internet". Por ello la globalización es un fenómeno básicamente económico, y que pauta lo político, lo cultural, las comunicaciones, es decir todo. Y tiene una relación muy estrecha o simbiótica con:

- Las telecomunicaciones, que reducen las distancias geográficas.

- La informática, tiene tal capacidad de condensación de conocimiento que nos hace perder el sentido de pasado, presente y futuro (es a histórica).

- En lo financiero, las más grandes operaciones se realizan en segundos. El circulante tiende a desaparecer para dar paso al dinero virtual (tarjetas de crédito). 
- El comercio mundial, se realiza por grandes bloques: UE, TLC, MERCOSUR, etc.

Este fenómeno económico propone la unificación de mercados, el dominio del capitalismo financiero y en el ámbito social se suceden características importantes como: la informática, a través del Internet comunicación sin fronteras. A Cristóbal Colon, le tomaron dos meses en llegar al Nuevo Mundo hoy se tarda solo unos segundos, y eso se debe a la tecnología llamada "internet", "comunicación en tiempo real". Como bien lo mencionaba Luis Palomino en una ponencia virtual:

La globalización nace como un concepto económico en un contexto político mundial. Internet nace como una necesidad infraestructural, pero deviene o se inserta en la dimensión cultural de las personas y las instituciones. Es fundamentalmente a través de Internet que se viabiliza dicha globalización.

Esto ha generado toda una problemática ambiental, que si bien es cierto viene desde la revolución industrial también es verdad que la globalización es un proceso y que en las últimas décadas se han incrementado como la "lluvia acida", problemas con la capa de ozono, "calentamiento global entre otras.

A nivel social hay cada vez más una agudización entre los ricos y pobres que genera desempleo masivo, a través de dos variables, la desmaterialización de la producción y la desproletarización.

A esto se debe sumar la presencia de la mujer en los espacios públicos, con cada vez más presencia laboral, y académica a esto se llama equidad de género.

El problema de la educación, que se podría resumir en lo que, el Center for Global Education, muestra en su página Web sobre los programas de estudios, la expresión "Lettheworld be yourclassroom!" Deja que el mundo sea tu aula.

\section{TENDENCIAS Y DESAFÍOS QUE PLANTEA LA GLOBALIZA- CIÓN:}

- El desarrollo vertiginoso e incontrolado de la ciencia y la tecnología ensancha las brechas socioeconómicas y hacen más 
asimétricas las relaciones entre los países más desarrollados y los países emergentes.

- Menores tendencias a la generación del empleo masivo. El empleo en general a pasado a ser un reflejo de la competitividad agregada de las empresas.

- Predominio de las políticas económicas, sobre las políticas sociales y "descalificación" del discurso político.

- Cambio de enfoques en el sistema educativo mundial, en el Perú sobre la base de los pilares: aprender a aprender, aprender a ser, aprender a hacer y aprender a convivir.

- Creciente preocupación mundial por el medio ambiente y por el mantenimiento equilibrado de todas las formas de vida. Mediante el desarrollo de nuevas actitudes en torno a la tesis del desarrollo humano y sostenible.

- Mayor protagonismo de las mujeres en el escenario internacional.

- Riesgo de pérdida de la diversidad cultural, como patrimonio universal, ante el avance monocultural.

\section{REFERENCIAS BIBLIOGRÁFICAS}

ABUSADA, Roberto y otros.

2000 La Reforma Incompleta, rescatando los noventa. Lima: Universidad del Pacifico.

ADORNO, Theodor; POPPER, Karl; HABERMAS, Jurgen, DAHRENDOR, Ralph; ALBERT, Hans; PILOT, Harold.

1973 La disputa del positivismo en la Sociología Alemana. Barcelona: Grijalbo.

AGAR, Michael.

1991 "Hacia un lenguaje etnográfico". En: Carlos Reynoso, El surgimiento de la antropología posmoderna, pp. 117-137. México, Gedisa Editorial.

ALDANA Rivera, Susana.

1999 Poderes de una región de frontera: comercio y familia en el norte (Piura, 1700-1830).- Lima: Panaca. 
AUGÉ, Marc y Jean-Paul COLLEYN. Qué es la antropología. Barcelona: 2005 Paidós Ibérica.

AUGÉ, Marc. Hacia una antropología de los mundos contemporáneos. 1996 Barcelona: Gedisa Editorial.

BOURDIEU, Pierre.

1993 Razones prácticas. Sobre la teoría de la acción. Barcelona, Editorial Anagrama. Cosas dichas. Barcelona: Gedisa Editorial.

BUNGE, Mario.

1982 Epistemología. La Habana: Editorial Ciencias Sociales.

1977 La Ciencia, su Método y su Filosofía. Buenos Aires: Siglo Veinte.

CARDOSO, Ciro F. S. y PÉREZ BRIGNOLI, H.

1976 Los métodos de la historia. Introducción a los problemas métodos $y$ técnicas de la historia demográfica, económica y social. Barcelona:Editorial Crítica.

CHÁVEZ ARELLANO, María Eugenia.

2000 "Antropología, economía y sociología. Un acercamiento epistemológico". En: Rafael PÉREZ TAYLOR et al., Aprender-comprender la antropología, pp. 273-300. México: CECSA.

CLIFFORD, James.

1991 "Introducción. Verdades parciales". En: J. CLIFFORD y G. E. MARCUS, Retóricas de la antropologia, pp. 25-60. Madrid: Júcar.

COMAS, José.

El 'fenómeno Fujimori' eclipsa a Vargas Llosa. Artículo publicado el 10 de abril de 1990 en el Diario El País.

CUCHE, Denys.

2002 La noción de cultura en las ciencias sociales. Buenos Aires: Nueva Visión.

DE SOUSA SANTOS, Boaventura.

2006 Conocer desde el Sur. Para una cultura política emancipatoria. Lima: UNMSM, Programa de Estudios sobre Democracia y Transformación Global, 285pp. 
DESCOLA, Philippe

2003 Antropología de la naturaleza. Lima: IFEA - Lluvia Editores, 2003

DURAND, Francisco. Backus y la desaparición de los apóstoles. En DES-

CO / Revista Quehacer Nro. 138 / Set. - Oct. 2002

DURKHEIM, Emile.

1969 Las Reglas del Método Sociológico. Buenos Aires: La Pléyade,

GEERTZ, Clifford.

2003 "Reflexiones antropológicas sobre temas filosóficos". Barcelona, Paidós Ibérica. 2005.

Descripción densa: hacia una teoría interpretativa de la cultura. En: Clifford GEERTZ, La interpretación de las culturas, pp. 19-40. Barcelona: Gedisa Editorial.

GIROUX, Sylvain y Ginette TREMBLAY

2004 Metodología de las ciencias humanas. La investigación en acción. México: Fondo de Cultura Económica.

GODELIER, Maurice.

1990 Lo ideal y lo material. Pensamiento, economías, sociedades. Madrid, Taurus Humanidades.

GONZALES DE OLARTE, Efraín

1998 El neoliberalismo a la peruana: economía política del ajuste estructural. 1990-1997. IEP. 146 pp.

GONZÁLEZ ECHEVARRÍA, Aurora

2006 "Epistemología y métodos en antropología: integración de métodos científicos y hermenéuticos y crítica epistemológica”, En: Revista de Antropología, PP. 11-40. Lima: UNMSM - FCS - EAPAN.

GOULDNER, Alvin.

Contra el Minotauro: el mito de una sociología libre de valores.

HABERMAS, Jürgen.

1988 La Lógica de las Ciencias Sociales. Madrid: Taurus.

HOBSWBAWN, Eric.

1991 Naciones y nacionalismo desde 1780. Madrid: Crítica. 
JIMÉNEZ, Félix.

Neoliberalismo y Republicanismo. Artículo publicado el 14 de septiembre de 2013 en el diario La Primera.

KONIG, René.

El problema de los Juicios de Valor en Max Weber. En PARSONS,

KOSELLECK, Reinhart

2004 Historia, Madrid: Editorial Trota, S.A.

KULA, Witold

1977 Problemas y métodos de la Historia económica. Barcelona: Ediciones Península.

LLOBERA, Josep R

1980 Hacia una historia de las ciencias sociales. Barcelona: Editorial nagrama.

LOMBARDI, Miguel

1991 Fundamento de Sociología. Separata UNMSM.

LÓPEZ, Sinesio

1991 El Dios Mortal, estado, sociedad y política en el Perú del siglo XX. Lima: Instituto Democracia y Socialismo.

LOWY, Michael

1972 Sobre el Método Marxista. Barcelona: Grijalbo.

MATOS MAR, José

1984 Desborde popular y crisis del estado: el nuevo rostro del Perú en la década de 1980. Instituto de Estudios Peruanos - IEP.

MOMMSEN, Wolfgang. Max Weber

1981 Sociedad e Historia. Barcelona: Editorial Alfa.

MORÓN, Eduardo

Homenaje a Jorge Camet. Artículo publicado el 02 de noviembre de 2013 en el diario El Comercio. 
PALTI, Elías José

2006 La nación como problema, los historiadores y la "cuestión nacional”, Buenos Aires: Fondo de Cultura Económica.

PARSONS, Talcott

Evaluación y objetividad en el ámbito de las Ciencias Sociales.

Una interpretación de los trabajos de Max Weber. En PARSONS, Presencia de Max Weber.

TANAKA, Martín, Patricia ZÁRATE y Ludwig HUBER.

2011 Mapa de la conflictividad social en el Perú. Análisis de sus principales causas. Lima: PCM - COMISIÓN EUROPEA - PNUD.

2013 Alberto Fujimori. Artículo publicado el 27 de octubre, diario La República.

TOWNSEND K., Augusto

2013 Cuando le hicimos caso a Margaret Thatcher. Artículo publicado el 15 de abril, diario El Comercio.

TUESTA, Fernando

1995 El Fenómeno Fujimori. Artículo publicado el 13 de abril, diario El Mundo. Una interpretación de los trabajos de Max Weber. En PARSONS, Presencia de Max Weber.

WEBER, Max

1973 Ensayos sobre Metodología Sociológica. Buenos Aires: Amorrortu.

WHITE, Hayden

2003 El texto histórico como artefacto literario. España: Ediciones Paidós.

ZUBIZARRETA, Armando

1983 La aventura del trabajo intelectual. Cómo estudiar e investigar, $2^{\mathrm{a}}$ ed. Washington: Fondo Educativo Interamericano.

\section{ARTÍCULOS WEB}

LÓPEZ, Sinesio. El paso del Estado oligárquico al Estado neoliberal. Apéndice sobre la tercera incursión democratizadora. Artículo del 
04/07/2009 http://erichluna.wordpress.com/2009/07/04/el-pasodel-estado-oligarquico-al-estado-neoliberal-6-apendice-sobre-latercera-incursion-democratizadora/

JIMÉNEZ Jaimes, Félix. El modelo Neoliberal Peruano: ¿Continuidad o Ruptura? Publicado en su Blog el lunes, 07 de junio 2009 http:// felixjimenez.blogspot.com/2009/06/el-modelo-neoliberal-peruano. html

FUENTES, Kriss. 5 de abril de 1992: Los 20 hechos más importantes que desencadenó el autogolpe de Alberto Fujimori. Publicado en el diario La República digital, el 05 de abril de 2012 http://www. larepublica.pe/05-04-2012/los-20-hechos-mas-importantes-quedesencadeno-el-autogolpe-de-alberto-fujimori

POLLAROLO G, Pierina. La Reforma del Estado. Publicada en noviembre 2005 http://www2.congreso.gob.pe/sicr/cendocbib/con4_uibd.nsf /162FDDB1B9FD35CF05257C00005ACBB3/\$FILE/3_agenda pendiente_en_modernizacion_del_estado.pdf 